\title{
Stability of a localized and greedy routing algorithm
}

\author{
Christelle Caillouet Florian Huc \\ Drakkar, LIG Lab TCS-SENSOR Lab, \\ Nicolas Nisse, Stéphane Pérennes \\ Hervé Rivano \\ Grenoble, France University of Geneva, Suisse \\ CNRS-INRIA-UNS Mascotte \\ Sophia Antipolis, France \\ CNRS-INRIA-UNS Mascotte \\ INSA Lyon, CITI, France
}

\begin{abstract}
In this work, we study the problem of routing packets between undifferentiated sources and sinks in a network modeled by a multigraph. We consider a distributed and local algorithm that transmits packets hop by hop in the network and study its behavior. At each step, a node transmits its queued packets to its neighbors in order to optimize a local gradient. This protocol is greedy since it does not require to record the history about the past actions, and localized since nodes only need information about their neighborhood.

A transmission protocol is stable if the number of packets in the network does not diverge. To prove the stability, it is sufficient to prove that the number of packets stored in the network remains bounded as soon as the sources inject a flow that another method could have exhausted. The localized and greedy protocol considered has been shown to be stable in some specific cases related to the arrival rate of the packets. We investigate its stability in a more general context and therefore reinforce results from the literature that worked for differentiated suboptimal flows.

We show that, to prove the stability of this protocol, it is sufficient to prove the intuitive following conjecture: roughly, if the protocol is stable when all sources inject the maximum number of packets at each turn and no packets are lost, then the protocol is stable whatever be the behavior of the network (i.e., when less packets are injected and some of them may be lost).
\end{abstract}

\section{INTRODUCTION}

The actual progress of networks involves an increasing interest for distributed algorithms that use only few information about the network [1]. We study a localized protocol for routing packets dynamically. We study the protocol stability region, i.e., the packet arrival rates such that the number of packets stored at the nodes of the network remains bounded.

In previous works, Srikant et al.[2] studied distributed and localized algorithms to transmit packets in a network. In their study, they do not deal with the routing and only focus on the call scheduling for one-hop communications when calls are matching and packets enter the network continuously. They base their work on an article by Tassioulas et al. [3] who have proposed a family of stable algorithms. In both of these cases, packets are injected into the network following a stochastic process that respects a strict feasibility constraint, meaning that the number of added packets at each time is always strictly lower than the value of the maximum flow.

Other works have considered processes in which packets are generated by an adversary who wants to make the protocol fail [4]. Two distributed algorithms in dynamic networks in which topology and traffic settings can change among time have been developed [5]. In that case, the proof of stability has been done for networks with only one destination node.

In this work, we consider a simplified network model in which sources inject packets into the network, then the nodes forward these packets according to a local greedy gradient computation with the only information of their neighbors' state, and sinks extract the packets from the network. This behavior can be related to the distributed algorithm for the maximum flow problem proposed by Goldberg and Tarjan [6].

We show that, when running in a network in which a set of undifferentiated sources injects packets to be forwarded to a set of sinks, the aforesaid protocol is stable on an optimal region if the following intuitive conjecture is correct. More precisely, we show under Conjecture 1 that when the number of packets generated could have been exhausted by any other method, the number of packets stored in the network remains bounded. Roughly, Conjecture 1 assumed in a specific case claims that if the protocol is stable when all sources inject the maximum number of packets at each turn and no packets are lost, then the protocol is stable whatever be the behavior of the network (see Section V).

\section{Model AND DEFInitions}

Let $G=(V, E)$ be a multigraph modeling the considered network. We denote by $\Delta$ the maximum degree of $G$ : $\Delta=\max _{v \in V}|\Gamma(v)|$, where $\Gamma(u)$ is the neighborhood of $u \in V$. Let $\mathcal{S} \subseteq V$ and $\mathcal{D} \subseteq V$ be respectively the sets of source and destination nodes. To each source $s \in \mathcal{S}$, resp., destination $d \in \mathcal{D}$, is associated an integer $\operatorname{in}(s)>0$, resp., out $(d)>0$. To each vertex is associated a queue length which represents the number of packets waiting to be transmitted at this node. We represent this queue length by $q_{t}(v)$ for $v \in V$ and a given time step $t$. Such a network is called $\mathcal{S}$-D-network, depicted on Fig. 1.

The network is synchronous and at each time step:

- each source $s \in \mathcal{S}$ injects $i n(s)$ packets in its queue,

- each link can transmit at most 1 packet, and this packet can be lost without any notification,

- each sink $d \in \mathcal{D}$ extracts $\min \left\{\right.$ out $\left.(d), q_{t}(d)\right\}$ packets of its queue. 
All links can transmit at the same time, so we do not consider interference constraints. We denote by $E_{t}$ the set of links that transmit a packet at time $t$.

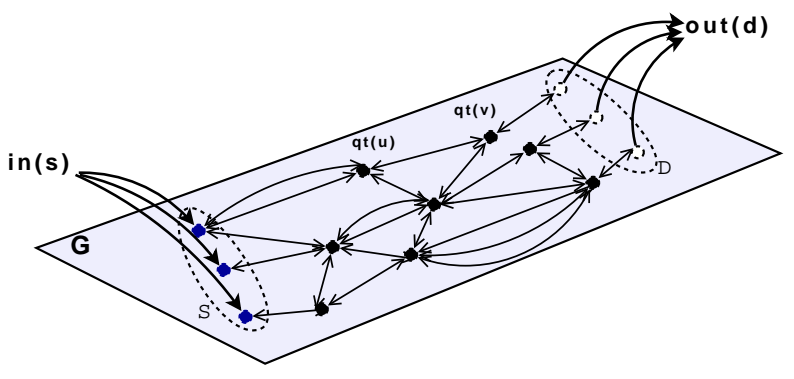

Fig. 1. The multigraph $G$ representing the network.

The arrival rate of the $\mathcal{S}$ - $\mathcal{D}$-network is defined as the sum of packets injected in the source's queues at a given time step: $\sum_{s \in \mathcal{S}} i n(s)$. We are interested in the total number of stored packets at a given time in the network. We quantify this number in the following definition:

Definition 1 ( $\mathcal{S}$-D -network state): The network state at time $t$ is defined by the function $P_{t}=\sum_{v \in V} q_{t}^{2}(v)$.

\section{A. The $L G G$ protocol}

The $\mathcal{S}$-D -network nodes run Algorithm 1 simultaneously. They only need to get access to the queue length of their neighbors.

At each time step $t$, each source $s$ injects $i n(s)$ packets in its queue. Then, each node $u$ transmits 1 packet through each of its outgoing arcs $u v$ if $v$ has a smaller queue length, as long as $u$ still has packets in its queue. In particular, if $u$ has more than $q_{t}(u)$ neighbors with smaller queue length, then it chooses to send to its $q_{t}(u)$ neighbors of smallest queue length. This choice has no impact on the system stability. The set of transmissions of $u$ at time $t$ is denoted by $E_{t}(u)$, i.e, $E_{t}(u)$ represents the set of links incident to $u$ transmitting a packet from $u$ at time $t$. Let $\cup_{u \in V} E_{t}(u)=$ $E_{t}$. Packets directed to node $v$ are deleted from $u$ 's queue, and, for each successful transmission, 1 packet is added to $v$ 's queue (recall that transmission may fail). Finally, each sink $d$ removes $\min \left\{\right.$ out $\left.(d), q_{t}(d)\right\}$ packets from its queue and step $t$ is over.

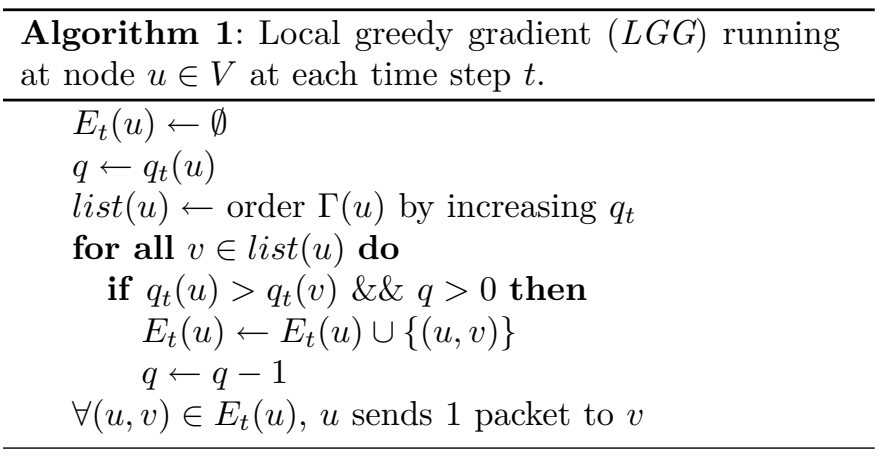

To study the behavior of LGG on a $\mathcal{S}$-D-network $G$, we define the notion of stability which we want to guaranty:
Definition 2 (Stability): Given an execution of LGG in a $\mathcal{S}$-D-network $G$, LGG is stable on $G$ if the number of packets stored in any node remains bounded. Note that it is equivalent to the sequence $\left(P_{t}\right)_{t \in \mathbb{N}}$ being bounded.

We want to prove that LGG is stable on any $\mathcal{S}$ - $\mathcal{D}$ network $G$ as soon as the arrival rate is feasible. Some definitions and requirements about the arrival rate are now specified before entering the details of the formal proof.

\section{B. Stability: preliminaries}

Let $G^{*}$ be the multigraph obtained from $G$ by adding a virtual source $s^{*}$ and a virtual sink $d^{*}$, with a link of capacity in $(s)$ between $s^{*}$ and $s$ for all $s \in \mathcal{S}$, and a link of capacity out $(d)$ between $d$ and $d^{*}$ for all $d \in \mathcal{D}$ (Fig. 2).

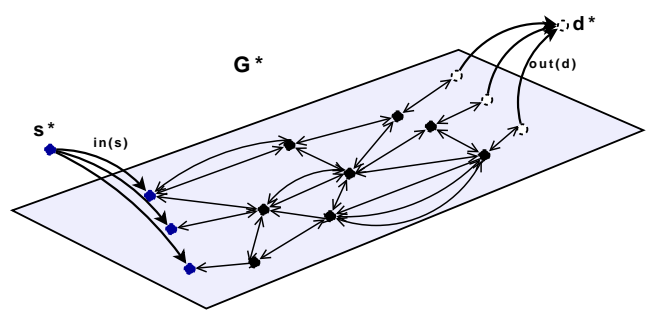

Fig. 2. The multigraph $G^{*}$ extending the $\mathcal{S}$ - $\mathcal{D}$-network $G$.

In the extended graph $G^{*}$, we consider a flow $\Phi$ from $s^{*}$ to $d^{*}$ verifying the following constraints:

$$
\begin{aligned}
& \text { - } \Phi(e) \leqslant c(e)= \begin{cases}1 & \forall e \in E(G) \\
\operatorname{out}(d) & \forall e=\left(d, d^{*}\right) \\
\operatorname{in}(s) & \forall e=\left(s^{*}, s\right)\end{cases} \\
& \text { - } \sum_{e \in \Gamma^{+}(v)} \Phi(e)=\sum_{e \in \Gamma^{-}(v)} \Phi(e), \forall v \in V(G) .
\end{aligned}
$$

Such a flow in $G^{*}$ is said feasible with value $f(\Phi)=$ $\sum_{e=\left(s^{*}, s\right)} \Phi(e)=\sum_{e=\left(d, d^{*}\right)} \Phi(e)$. We use the existence of a feasible flow with value greater than or equal to the arrival rate in LGG to define the feasibility of a $\mathcal{S}$ - $\mathcal{D}$-network. In particular, given capacities $i n(s)$, the flow must saturate links $\left(s^{*}, s\right)$.

Definition 3 (Feasible $\mathcal{S}$-D -network): A $\mathcal{S}$-D -network $G$ is feasible if it exists a $s^{*}-d^{*}$-flow $\Phi$ in $G^{*}$ such that $i n(s)=$ $\Phi\left(s^{*}, s\right)$ for each source $s \in \mathcal{S}$.

If a $\mathcal{S}$-D-network is feasible, the value of a maximum $s^{*}$ $d^{*}$-flow $\Phi$ given the link capacities $i n(s)$ is equal to the arrival rate $\sum_{s \in \mathcal{S}} i n(s)$. But increasing the capacity of links $\left(s^{*}, s\right)$ may allow a feasible flow in this modified $G^{*}$. In the following, we denote $f^{*}$ the value of a maximum $s^{*}-d^{*}$ flow in $G^{*}$ with infinite capacity on links $\left(s^{*}, s\right)$, therefore $\sum_{s \in \mathcal{S}} i n(s) \leqslant f^{*}$. In order to compare the performances of our algorithm LGG with an optimal algorithm consisting in sending the packets through the links of a maximum flow, we consider two cases: the difference between the maximum flow and the arrival rate is either equal to 0 or strictly positive.

Definition 4 (Unsaturated $\mathcal{S}$-D-network): A feasible $\mathcal{S}$ $\mathcal{D}$-network $G$ is unsaturated if it exists a fractional $s^{*}$ $d^{*}$-flow $\Phi$ in $G^{*}$ in which the capacity of links $\left(s^{*}, s\right)$ is 
increased to $(1+\epsilon) \operatorname{in}(s)$ for some $\epsilon>0$ at each source $s \in \mathcal{S}$. Otherwise, the network is saturated.

In other words, a $\mathcal{S}$-D-network $G$ is unsaturated if its arrival rate is strictly feasible, corresponding to the stability region defined by Tassiulas and Ephremedis in the general case of a multi-commodity flow [3].

In the rest of the paper, we prove the following theorem is true if Conjecture 1 is valid (Conjecture 1 is stated in details in Section V.):

Theorem 1: Let $G$ be a $\mathcal{S}$-D-network. If $G$ is feasible, then the protocol LGG is stable on $G$. Otherwise, the number of packets stored in the network may diverge with time no matter what algorithm is used.

Regardless to Conjecture 1, remark first that the system may diverge if $\sum_{s \in \mathcal{S}} i n(s)>f^{*}$. Indeed, without any assumptions on the packet loss, we can assume that none are lost. Then, looking at a $\mathcal{S}-\mathcal{D}$ minimum cut $(A, B)$ (of value $\left.f^{*}\right)$, with $\mathcal{S} \subseteq A$, at each step at most $f^{*}$ packets leave $A$ for $B$ whereas $\sum_{s \in \mathcal{S}} i n(s)\left(>f^{*}\right)$ enter it. So $P_{t}$ increases at each step.

In the following, we omit the adjective feasible when considering a $\mathcal{S}$ - $\mathcal{D}$-network $G$, as it is a prerequisite for the proof of the theorem.

The remainder of this paper is organized as follows. In the next section, we prove Theorem 1 for an unsaturated $\mathcal{S}$-D-network. Then we introduce in Section IV an extended network model to deal with the proof of Theorem 1 in the general case, covering the case of a saturated $\mathcal{S}-\mathcal{D}$ network in Section V.

\section{Stability of AN Unsaturated $\mathcal{S}$ - $\mathcal{D}$-NETWORK}

Lemma 1: If the $\mathcal{S}$-D-network $G$ is unsaturated, then the network state $P_{t}$ is upper bounded by a constant depending only on the network and the arrival rate.

To prove this lemma, we first show that the network state evolution between two consecutive time steps is upper bounded. Then, we prove that, if the network state is sufficiently large at some time step, then it decreases significantly at the next time step. These two properties allow to derive an upper bound for the network state for all $t$, leading to the stability of the protocol LGG on $G$.

Property 1: The growth of the network state between two consecutive steps stays bounded: $\forall t, P_{t+1}-P_{t} \leqslant 5 n \Delta^{2}$.

Proof: $G$ is unsaturated, so by definition it exists a flow $\Phi$ from $s^{*}$ to $d^{*}$ in $G^{*}$ such that, for all source $s \in \mathcal{S}$, $\operatorname{in}(s)<\Phi\left(s^{*}, s\right)$.

Let us consider the evolution of the network state between time step $t$ and $t+1$ :

$$
\begin{aligned}
P_{t+1}= & \sum_{v \in V} q_{t+1}^{2}(v) \\
= & \sum_{u \in V} q_{t}^{2}(v)+\sum_{v \in V}\left(q_{t+1}(v)-q_{t}(v)\right)^{2} \\
& +2 \sum_{v \in V} q_{t}(v)\left(q_{t+1}(v)-q_{t}(v)\right) .
\end{aligned}
$$

All links of $G$ have capacity 1 . So, for all $v \in V$, $\left(q_{t+1}(v)-q_{t}(v)\right) \leq \Delta$, where $\Delta$ is the maximum degree of $G$. By setting $\delta_{t}=\sum_{v \in V} q_{t}(v)\left(q_{t+1}(v)-q_{t}(v)\right)$, we obtain:

$$
P_{t+1} \leqslant P_{t}+2 \delta_{t}+n \Delta^{2} \text {. }
$$

Equivalently, $\delta_{t}$ can be defined in function of the links in $E_{t}$ used by LGG at time step $t$ for the transmissions. In the following, $e=(u, v) \in E_{t}$ is oriented to indicate that the packet goes from $u$ to $v$. Then, $\delta_{t}$ becomes:

$$
\begin{aligned}
\delta_{t}= & \sum_{s \in \mathcal{S}} q_{t}(s) \operatorname{in}(s)+\sum_{(u, v) \in E_{t}}\left(q_{t}(v)-q_{t}(u)\right) \\
& -\sum_{d \in \mathcal{D}} q_{t}(d) \min \left\{\text { out }(d), q_{t}(d)\right\} .
\end{aligned}
$$

We now compare the variation of $P_{t}$ during an execution of LGG to the one obtained by pushing the packets along the paths allowing a maximum flow. Let us consider the set of paths between the sources $\mathcal{S}$ and the sinks $\mathcal{D}$ used by flow $\Phi$, and $E_{t}^{\Phi}$ the set of links (source-to-destination oriented) of these paths selected at time $t$. By summing the difference of the potential on each hop along these paths, we get:

$\sum_{(u, v) \in E_{t}^{\Phi}}\left(q_{t}(v)-q_{t}(u)\right)=-\sum_{s \in \mathcal{S}} q_{t}(s) \Phi\left(s^{*}, s\right)+\sum_{d \in \mathcal{D}} q_{t}(d) \Phi\left(d, d^{*}\right)$.

Let us now study the sum of the difference of the potential on the links used by LGG:

$\sum_{(u, v) \in E_{t}}\left(q_{t}(v)-q_{t}(u)\right)=\sum_{(u, v) \in E_{t}^{\Phi}}\left(q_{t}(v)-q_{t}(u)\right)-$
$\sum_{(u, v) \in E_{t}^{\Phi} \backslash E_{t}}\left(q_{t}(v)-q_{t}(u)\right)+\sum_{(u, v) \in E_{t} \backslash E_{t}^{\Phi}}\left(q_{t}(v)-q_{t}(u)\right)$.

By definition of LGG, for all $e=(u, v) \in E_{t}, q_{t}(v)-$ $q_{t}(u)<0$. So, $\sum_{(u, v) \in E_{t} \backslash E_{t}^{\Phi}}\left(q_{t}(v)-q_{t}(u)\right)<0$. Moreover if $e=(u, v) \in E_{t}^{\Phi} \backslash E_{t}$, then, again by definition of LGG, either $q_{t}(v) \geq q_{t}(u)$ or $q_{t}(u) \leq \Delta$. Indeed if $q_{t}(v)<q_{t}(u)$, our algorithm must send 1 packet from $u$ to $v$, unless $u$ has already sent all its available packets in $q_{t}(u)$. So:

$$
\sum_{(u, v) \in E_{t}^{\Phi} \backslash E_{t}}\left(q_{t}(v)-q_{t}(u)\right) \geqslant \sum_{(u, v) \in E_{t}^{\Phi} \backslash E_{t}}(-\Delta) \geqslant-n \Delta^{2}
$$

and $\sum_{(u, v) \in E_{t}}\left(q_{t}(v)-q_{t}(u)\right) \leqslant-\sum_{s \in \mathcal{S}} q_{t}(s) \Phi\left(s^{*}, s\right)+$ $\sum_{d \in \mathcal{D}} q_{t}(d) \Phi\left(d, d^{*}\right)+n \Delta^{2}$.

From equation 3 , we deduce that for all $t$ :

$$
\begin{aligned}
\delta_{t} & \leqslant \sum_{s \in \mathcal{S}} q_{t}(s)\left(\operatorname{in}(s)-\Phi\left(s^{*}, s\right)\right) \\
& +\sum_{d \in \mathcal{D}} q_{t}(d)\left(\Phi\left(d, d^{*}\right)-\min \left\{\text { out }(d), q_{t}(d)\right\}\right)+n \Delta^{2}
\end{aligned}
$$

As the network is unsaturated, by definition of $\Phi$, in $(s)<\Phi\left(s^{*}, s\right)$ for all $s \in \mathcal{S}$. The sum of the queue lengths on the sources of $G$ contributes negatively to the upper bound of $\delta_{t}$. So we can neglect it. The same happens with the sum on the sinks if either $\min \left\{\operatorname{out}(d), q_{t}(d)\right\}=q_{t}(d)$ 
and $\Phi\left(d, d^{*}\right) \leqslant q_{t}(d)$, or $\min \left\{\right.$ out $\left.(d), q_{t}(d)\right\}=\operatorname{out}(d)$. The latter case happens when $\min \left\{\operatorname{out}(d), q_{t}(d)\right\}=q_{t}(d)$ and $\Phi\left(d, d^{*}\right)>q_{t}(d)$. Since links of $G$ have capacity 1, $\Phi$ is bounded by $\Delta$, leading to $\sum_{d \in \mathcal{D}} q_{t}(d)\left(\Phi\left(d, d^{*}\right)-\right.$ $\left.\min \left\{\operatorname{out}(d), q_{t}(d)\right\}\right) \leqslant n \Delta^{2}$.

We finally obtain an upper bound for $\delta_{t}$ : $\delta_{t} \leq 2 n \Delta^{2}$. In particular, from Inequality 2 , we upper bound the difference of the network state between step $t+1$ and $t$ :

$$
P_{t+1}-P_{t} \leqslant 5 n \Delta^{2} \text {. }
$$

Let introduce $\epsilon=\min _{s \in \mathcal{S}}\left(\Phi\left(s^{*}, s\right)-i n(s)\right)$ which is strictly positive by definition of an unsaturated network.

Property 2: Let $Y=\left(\frac{5 n f^{*}}{\epsilon}+3 n\right) \Delta^{2}$. If $P_{t}$ is large, i.e. $P_{t}>n Y^{2}$, then at the next step, the number of stored packets in the network decreases: $P_{t+1}-P_{t}<-5 n \Delta^{2}$.

Proof: From Inequality 2 , the proof is equivalent to show that, if $P_{t}>n Y^{2}$, then $\delta_{t}<-3 n \Delta^{2}$. The rest of the proof is divided into two parts depending on the existence of a node with large queue length in the network.

Let us first assume that it exists a source $s \in \mathcal{S}$ such that $q_{t}(s) \geq \frac{5 n}{\epsilon} \Delta^{2}$. Then, using Inequality 5 and the unsaturated property of the network, we can upper bound $\delta_{t}$ and prove the first part of Property 2:

$$
\delta_{t} \leqslant-\epsilon q_{t}(s)+2 n \Delta^{2}<-3 n \Delta^{2} .
$$

In the second case, $q_{t}(s)<\frac{5 n}{\epsilon} \Delta^{2}$ for all $s \in \mathcal{S}$. If $P_{t} \geqslant$ $n Y^{2}$, then it exists $x \in V \backslash \mathcal{S}$ such that $q_{t}(x) \geq Y$. Let $x=$ $u_{1}, u_{2}, \cdots, u_{k}$ be a path from $x$ to $u_{k}$ such that $u_{k}=d \in \mathcal{D}$ (maybe $d=x$ ). Then: $\sum_{i<k, q_{t}\left(u_{i}\right)>q_{t}\left(u_{i+1}\right)}\left(q_{t}\left(u_{i+1}\right)-\right.$ $\left.q_{t}\left(u_{i}\right)\right)-q_{t}\left(u_{k}\right) \min \left\{\operatorname{out}\left(u_{k}\right), q_{t}\left(u_{k}\right)\right\} \stackrel{-}{\leqslant} q_{t}(x)$. This sum contributes negatively to $\sum_{(u, v) \in E_{t}}\left(q_{t}(v)-q_{t}(u)\right)-$ $\sum_{d \in \mathcal{D}} q_{t}(d) \min \left\{\right.$ out $\left.(d), q_{t}(d)\right\}$ (recall that the terms of the first part of the sum are negative since they are used by LGG). From equation 3, we thus obtain that:

$$
\begin{aligned}
\delta_{t} & \leqslant \sum_{s \in \mathcal{S}} q_{t}(s) \operatorname{in}(s)-q_{t}(x) \\
& <f^{*} \max _{s \in \mathcal{S}} q_{t}(s)-q_{t}(x) \leqslant f^{*} \frac{5 n}{\epsilon} \Delta^{2}-q_{t}(x) \leqslant-3 n \Delta^{2} .
\end{aligned}
$$

By injecting the bound of $\delta_{t}$ in Inequality 2 , we obtain a strict upper bound on the network state evolution:

$$
P_{t+1}-P_{t}<-5 n \Delta^{2} \text {. }
$$

From Properties 1 and 2 we deduce that, for all $t$, $P_{t} \leqslant n Y^{2}+5 n \Delta^{2}$ which bounds the number of packets stored in the network at each time step and prove the strict stability of our algorithm. We remark that the packet losses here only improve the protocol stability.

In the case of a saturated $\mathcal{S}$ - $\mathcal{D}$-network in which a flow with value $(1+\epsilon) \operatorname{in}(s)$ on each link $\left(s^{*}, s\right)$ is unfeasible, then the previous techniques do not permit to control the variations of the second derivative in Equation 1. In order to tackle these, we must generalize the network behavior before addressing the proof of stability by induction on the network size. This generalization is presented in the next section, through the definition of the $R$-generalized $\mathcal{S}$ - $\mathcal{D}$-networks.

\section{IV. $R$-GENERALIZED $\mathcal{S}$ - $\mathcal{D}$-NETWORKS}

Let $G=(V, E)$ be a feasible $\mathcal{S}$ - $\mathcal{D}$-network and let $(A, B)$ be a minimum cut in $G^{*}$, i.e. $(A, B)$ is a node partition of $G^{*}$ such that $s^{*} \in A, d^{*} \in B$, and the sum of the link capacities between $A$ and $B$ is minimum. In the case where $\mathcal{S} \subseteq A$ and $\mathcal{D} \subseteq B,(A, B)$ is called a $\mathcal{S}$-D -cut, an example is depicted in Fig. 3.

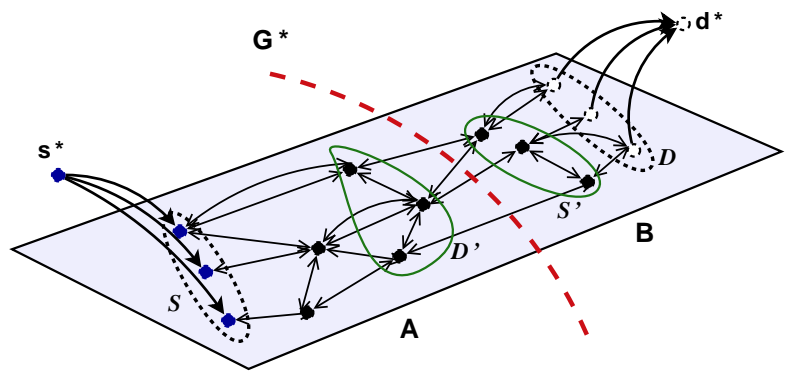

Fig. 3. A minimum $\mathcal{S}$ - $\mathcal{D}$-cut in the extended graph $G^{*}$ and associated subsets $\mathcal{S}^{\prime}$ and $\mathcal{D}^{\prime}$

The proof of Theorem 1 is done by induction on the network size $|V|$. To do so, we need to generalize the network to model the special behavior of the nodes located in the border of the cut $(A, B)$ and apply the induction hypothesis independently on $B$ and then $A$. We then define the $R$-generalized $\mathcal{S}$-D $\mathcal{D}$-networks, in which $R \geq 0$ is a constant, and such that every classical $\mathcal{S}$ - $\mathcal{D}$-network is a 0 -generalized $\mathcal{S}$ - $\mathcal{D}$-network in the generalized model. The purpose of the induction is to prove that, for all $R \geq 0$ and in any feasible $R$-generalized $\mathcal{S}$-D-network $G$, LGG is stable. In particular, this proves that LGG is stable in any $\mathcal{S}$ - $\mathcal{D}$-network

More precisely, we first prove that parts $B$ and then $A$ acts as $R$-generalized $\mathcal{S}$-D -networks for well chosen constants, allowing us to apply the induction hypothesis. Several cases must be considered, depending on the location of the links between $A$ and $B$ that can be in $G$, or incident to the virtual nodes $s^{*}$ and $d^{*}$ added in $G^{*}$. This latter case corresponds to our induction basis and is tackled in Sections V-A and V-B.

The generalization of the network behavior is needed when the cut $(A, B)$ is located inside $G$. First, we can remark that partition $B$ may be viewed as a particular case of a $\mathcal{S}^{\prime}$-D-network, in which $\mathcal{S}^{\prime}$ is the set of nodes in $B$ adjacent to a node in $A$ (Fig. 3). Each of these nodes $s^{\prime} \in \mathcal{S}^{\prime}$ corresponds to a source in $B$ that injects at most $\left|\Gamma_{\mid A}\left(s^{\prime}\right)\right|+i n\left(s^{\prime}\right)$ packets in its queue at each step, where $\Gamma_{\mid A}\left(s^{\prime}\right)$ represents the neighborhood of $s^{\prime}$ in $A$, and in $\left(s^{\prime}\right)>0$ in the case of $s^{\prime} \in \mathcal{S}$ in $G$. The hypothesis of the random packet losses validates the case when $s^{\prime}$ sends packets to a node that is located in partition $A$. 
Similarly, if $s^{\prime} \in \mathcal{D}$ is a destination that extracts some packets out of the network, then the extracted packets can be viewed as lost in the original network. In order to generalize the behavior, we define pseudo-sources that are less constrained than the previous classical sources.

Definition 5 (Pseudo-source): A pseudo-source s injects at most in $(s)>0$ packets in its queue at the beginning of each step.

In a second time, we suppose that the number of stored packets in $B$ is bounded by some constant $R$, and show that partition $A$ can also be viewed as a $\mathcal{S}$ - $\mathcal{D}^{\prime}$-network, in which $\mathcal{D}^{\prime}$ contains all nodes in $A$ that have at least one neighbor in $B$ (Fig. 3). Each $d^{\prime} \in \mathcal{D}^{\prime}$ has the following behavior: if the queue length of $d^{\prime}$ is high enough $\left(q_{t}\left(d^{\prime}\right)>R\right.$ for some constant $\left.R\right)$, then $d^{\prime}$ extracts at least $\min \left\{\left|\Gamma_{\mid B}\left(d^{\prime}\right)\right|+\operatorname{out}\left(d^{\prime}\right), q_{t}\left(d^{\prime}\right)-R\right\}$ packets of its queue (since $d^{\prime}$ is higher than all its neighbors in $B$ ). Moreover, since the nodes in $\mathcal{D}^{\prime}$ whose queue length is lower than $R$ may receive some packets from nodes in $B$, their behavior towards $A$ can be viewed as if they could hide some packets of their queue to the nodes in $A$. In other words, for each $d^{\prime} \in \mathcal{D}^{\prime}$ such that $q_{t}\left(d^{\prime}\right) \leqslant R, d^{\prime}$ may declare a queue length $q_{t}^{\prime}\left(d^{\prime}\right) \leqslant R$ to nodes in $A$, generalizing the behavior of the destination nodes.

Definition 6 (R-pseudo-destination): A generalized destination $d$ extracts at most out $(d)>0$ packets of its queue at the end of each step, and, given a constant of retention $R \geq 0$ :

(i) if $q_{t}(d)>R$, then $d$ extracts at least $\min \left\{\right.$ out $\left.(d), q_{t}(d)-R\right\}$ packets of its queue,

(ii) for each $u \in \Gamma(d), d$ reveals a queue size $q_{t}^{\prime}(d)$ defined as follows:

- if $q_{t}(d)>R$, then $d$ declares $q_{t}^{\prime}(d)=q_{t}(d)$,

- if $q_{t}(d) \leqslant R$, then $d$ declares a queue length $q_{t}^{\prime}(d) \leqslant R$.

We now combine these two definitions and define a $R$ generalized $\mathcal{S}$ - $\mathcal{D}$-network :

Definition 7 (R-generalized source/destination): Let

$R \geqslant 0$, a $R$-generalized node $v$ injects at most $i n(v)>0$ packets in its queue at the beginning of each step, extracts at most $\operatorname{out}(v)>0$ packets of its queues at the end of each step, and:

(i) if $q_{t}(d)>R$, then $d$ extracts at least $\min \left\{\right.$ out $\left.(d), q_{t}(d)-R\right\}$ packets of its queue,

(ii) for each $u \in \Gamma(d), d$ reveals a queue size $q_{t}^{\prime}(d)$ defined as follows:

- if $q_{t}(d)>R$, then $d$ declares $q_{t}^{\prime}(d)=q_{t}(d)$,

- if $q_{t}(d) \leqslant R$, then $d$ declares a queue length $q_{t}^{\prime}(d) \leqslant R$.

If $\operatorname{in}(v) \leqslant \operatorname{out}(v)$, then $v$ is called a $R$-generalized destination, otherwise it is a $R$-generalized source.

Definition 8 (R-generalized $\mathcal{S}$-D -network): A $R$-generalized $\mathcal{S}$-D -network is a multigraph $G$ containing a set $\mathcal{S}$ of $R$-generalized sources, and a set $\mathcal{D}$ of $R$-generalized destinations. All the other nodes of $G(v \in V \backslash(\mathcal{S} \cup \mathcal{D}))$

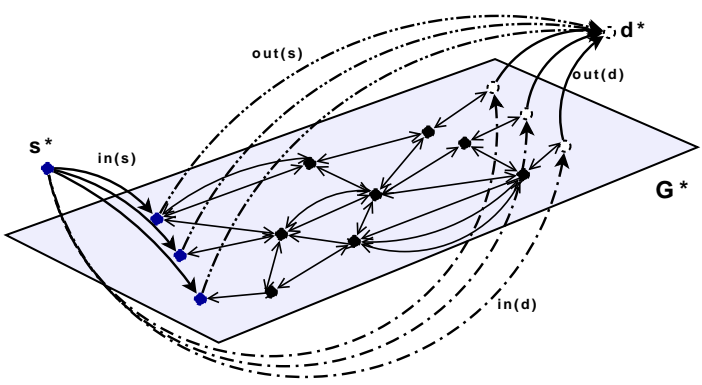

Fig. 4. An extended $R$-generalized $\mathcal{S}$-D-network $G^{*}$.

keep their "classical" behavior, i.e. the same as in the $\mathcal{S}$ - $\mathcal{D}$-network defined in Section II.

Remark 1: Every node $v$ of the network that is not in $\mathcal{S} \cup \mathcal{D}$ is set with $\operatorname{in}(v)=\operatorname{out}(v)=0$. Nevertheless, these values may change during the induction process, and then $v$ may become a $R$-generalized source or destination.

A $\mathcal{S}$-D $\mathcal{D}$-network is clearly a 0 -generalized $\mathcal{S}$ - $\mathcal{D}$-network. Indeed, from definition 7, 0-generalized sources and destinations have the following properties:

- a source $s$ injects at most in $(s)$ packets in its queue at the beginning of each step,

- a destination $d$ extracts at most out $(d)$ packets, and at least $\min \left\{\right.$ out $\left.(d), q_{t}(d)\right\}$ packets of its queue at the end of each step (since $R=0$ brings us in the $(i)$ 's case of definition 7$)$. It never lies on its queue length since $q_{t}(d)$ is always greater than or equal to 0 , so greater to $R$.

Packet losses are modeled by the ability of a source $s$ to inject less than in $(s)$ in the network. In return, the behavior is the same as in a $\mathcal{S}$ - $\mathcal{D}$-network.

We define an extended generalized network $G^{*}$ as in Section II with virtual nodes $s^{*}$ and $d^{*}$ added to $G$, and with links $\left(s^{*}, v\right)$ and $\left(v, d^{*}\right)$ of capacity $i n(v)$ and out $(v)$ respectively, $\forall v \in \mathcal{S} \cup \mathcal{D}$. Similarly to Definitions 3 and 4 , we say that $G$ is feasible if it exists a feasible flow $\Phi$ in $G^{*}$ with $\Phi\left(s^{*}, v\right)=i n(v), \forall v \in \mathcal{S} \cup \mathcal{D}$ (Figure 4). A $R$-generalized $\mathcal{S}$-D $\mathcal{D}$-network $G$ is unsaturated if it exists a feasible $s^{*}-d^{*}$-flow $\Phi$ in $G^{*}$ in which links $\left(s^{*}, v\right)$ have capacity $(1+\epsilon) \operatorname{in}(v), \forall v \in \mathcal{S} \cup \mathcal{D}$.

\section{Stability of a $R$-Generalized $\mathcal{S}$ - $\mathcal{D}$-NeTwork}

This section is devoted to the proof of stability of our algorithm LGG on a feasible $R$-generalized $\mathcal{S}$ - $\mathcal{D}$-network, under the following conjecture:

Conjecture 1: If our protocol is stable in a feasible $R$ generalized $\mathcal{S}$ - $\mathcal{D}$-network in which the generalized sources $s \in \mathcal{S}$ injects exactly in $(s)$ packets in their queue at each step, and when no packet loss is allowed, then LGG is stable in any feasible $R$-generalized $\mathcal{S}$ - $\mathcal{D}$-network.

It is indeed intuitive to think that the packet generation process follows a domination scheme: removing some packets should not lead to divergence. If the sequence $i n_{t}(v)$ is greater than another sequence $i n_{t}^{\prime}(v)$ at each time $t$ and 
for all node $v \in \mathcal{S} \cup \mathcal{D}$, then the state of the system should be lower using $i n_{t}^{\prime}$ than $i n_{t}$. Subject to the correctness of this conjecture, we prove the following theorem:

Theorem 2: For all constant $R \geqslant 0$, and in any feasible $R$-generalized $\mathcal{S}$ - $\mathcal{D}$-network $G$, the protocol LGG is stable. Moreover, LGG is stable in any feasible $\mathcal{S}$ - $\mathcal{D}$-network.

This theorem is a rephrasing of Theorem 1 in order to prove the stability of LGG in the saturated $R$-generalized $\mathcal{S}$-D-networks, for all $R \geqslant 0$. As we showed in the previous section that a $\mathcal{S}$ - $\mathcal{D}$-network is a 0 -generalized $\mathcal{S}$ $\mathcal{D}$-network, the proof of stability of LGG for the saturated $\mathcal{S}$ - $\mathcal{D}$-networks will be complete.

Let us consider a feasible $R$-generalized $\mathcal{S}$ - $\mathcal{D}$-network $G=(V, E)$, with $R \geqslant 0$. If $|V|=1$, our protocol is obviously stable. Suppose now that $|V|>1$. Let $\Phi$ be a maximum $s^{*}$-d $d^{*}$-flow in $G^{*}$ such that $\operatorname{in}(v)=\Phi\left(s^{*}, v\right)$, $\forall v \in \mathcal{S} \cup \mathcal{D}$, and let $(A, B)$ be a minimum cut in $G^{*}$ of value $|(A, B)|=\sum_{v \in \mathcal{S} \cup \mathcal{D}} i n(v)$. We then have three cases:

1) such a cut $(A, B)$ is unique and corresponds to $\left(\left\{s^{*}\right\},\left(V \cup\left\{d^{*}\right\}\right) \backslash\left\{s^{*}\right\}\right)$ : we prove in Section V-A that $G$ is unsaturated, and that our protocol is stable by an adaptation of the proof of Section III,

2 ) one single other cut exists and corresponds to $\left(\left(V \cup\left\{s^{*}\right\}\right) \backslash\left\{d^{*}\right\},\left\{d^{*}\right\}\right)$ : we prove in Section V-B the stability of LGG subject to the correctness of Conjecture 1,

3 ) it exists such a cut $(A, B)$ in $G$ : we prove in Section $\mathrm{V}-\mathrm{C}$ the stability by induction on the size of $G$, as introduced at the beginning of the previous section.

Cases 1 and 2 correspond to the basis of our induction.

\section{A. Unsaturated $R$-generalized $\mathcal{S}$-D -network}

There is an unique minimum cut $(A, B)$ in $G^{*}$ with $A=\left\{s^{*}\right\}$, this means that the flow is only constrained by the amount of packets injected by the virtual source $s^{*}$, i.e. by the capacities $i n(v)$ on links $\left(s^{*}, v\right), \forall v \in \mathcal{S} \cup \mathcal{D}$. Then, there is a constant $\epsilon>0$ allowing a feasible flow $\Phi$ in $G^{*}$ in which the arrival rate is $(1+\epsilon) i n(v)$ at each node $v \in \mathcal{S} \cup \mathcal{D}$. Otherwise, it exists another cut between the saturated source and the destinations with value equal to the one of the unique minimum cut $(A, B)$, leading to a contradiction. This is therefore the definition of an unsaturated network as defined for a classical $\mathcal{S}$ - $\mathcal{D}$-network in Section II.

In this context, Lemma 1 becomes:

Lemma 2: Given an unsaturated $R$-generalized $\mathcal{S}$ - $\mathcal{D}$ network $G$ running LGG, the network state $P_{t}$ is upper bounded for all $t$.

This lemma can be proved by adapting the proof of Lemma 1. It is decomposed into two properties bounding the difference of the network state between two consecutive steps. The first property which upper bounds the growth of the network state becomes:
Property 3: The growth of the network state between two consecutive steps remains bounded for all $t$ :

$$
\begin{aligned}
P_{t+1}-P_{t} \leqslant \quad & 2|\mathcal{S} \cup \mathcal{D}|\left(R+\text { out }_{\text {max }}\right) \text { out }_{\text {max }} \\
& +\Delta^{2}(3 n-2|\mathcal{S} \cup \mathcal{D}|)+4|\mathcal{S} \cup \mathcal{D}| \Delta R,
\end{aligned}
$$

where out max $_{\max }=\max _{v \in \mathcal{S} \cup \mathcal{D}}$ out $(v)$.

The second property needed to prove the stability of LGG on the unsaturated $R$-generalized $\mathcal{S}$ - $\mathcal{D}$-network is:

Property 4: Given a constant $Y$ large enough, if $P_{t}>$ $n Y^{2}$, at step $t+1$, the number of stored packets in the network strictly decreases:

$$
\begin{aligned}
P_{t+1}-P_{t}< & -2|\mathcal{S} \cup \mathcal{D}|\left(R+\text { out }_{\max }\right) \text { out }_{\max } \\
& -\Delta^{2}(3 n-2|\mathcal{S} \cup \mathcal{D}|)-4|\mathcal{S} \cup \mathcal{D}| \Delta R,
\end{aligned}
$$

where out max $_{\text {max }}=\max _{v \in \mathcal{S} \cup \mathcal{D}}$ out $(v)$.

The proof of these two properties is similar to the ones in Section III and can be found in a research report available online[7] and copied in the annex for the reviewers.

From Properties 5 and 6 , we conclude that, for all $t$, the network state $P_{t}$ is upper bounded, which limits the number of stored packets in the network at any time step and validates the stability of LGG in an unsaturated $R$ generalized $\mathcal{S}$-D $D$-network.

\section{B. $R$-generalized $\mathcal{S}$-D-network saturated at virtual node $d^{*}$}

We suppose here that $i n_{t}(v)=i n(v), \forall v \in \mathcal{S} \cup \mathcal{D}$ and $t$, and there is no packet loss. We prove the stability of LGG in this particular case. Conjecture 1 allows us to conclude that LGG is stable in the more general case with $i n_{t}(v) \leqslant i n(v)$ and possible packet losses.

In this case, $\left(\left\{s^{*}\right\},\left(V \cup\left\{d^{*}\right\}\right) \backslash\left\{s^{*}\right\}\right)$ is not the only minimum cut in $G^{*}$ : a second cut exists and is located at the virtual destination $d^{*}:(A, B)=\left(\left(V \cup\left\{s^{*}\right\}\right),\left\{d^{*}\right\}\right)$. In other words, $B$ contains only $d^{*}$. The value of the cut is $|(A, B)|=\sum_{v \in \mathcal{S} \cup \mathcal{D}} i n(v)=\sum_{v \in \mathcal{S} \cup \mathcal{D}}$ out $(v)$. There are two cases to be considered.

First, we assume that there is a time step $t_{0}$ and a

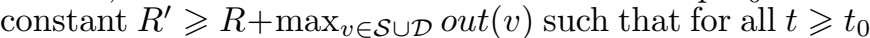
and $v \in \mathcal{S} \cup \mathcal{D}, q_{t}(v) \geqslant R^{\prime}$. From the definition of the $R$ generalized nodes, if $q_{t}(v)>R+\operatorname{out}(v)$, then $v$ extracts exactly out $(v)$ packets. At each step $t \geqslant t_{0}$, the arrival rate in LGG is lower than or equal to the extracting rate of the $R$-generalized sources/destinations, the growth of the number of stored packets is thus:

$$
\begin{aligned}
\sum_{v \in V} q_{t+1}(v) & =\sum_{v \in V} q_{t}(v)-\sum_{v \in V} \operatorname{out}(v)+\sum_{v \in V} \operatorname{in}(v) \\
& \leqslant \sum_{v \in V} q_{t}(v) \leqslant \sum_{v \in V} q_{t_{0}}(v)
\end{aligned}
$$

The network state is therefore bounded over time.

Then, we suppose that there is at least a node $v \in \mathcal{S} \cup \mathcal{D}$ infinitely bounded according to the following definition:

Definition 9 (Infinitely bounded node): A node is infinitely bounded if there is a constant such that its queue length goes below this constant an infinite number of times. More formally, a node $v \in V$ is infinitely bounded if $\exists M>0$ such that $\forall t_{0}, \exists t \geqslant t_{0}$ such that $q_{t}(v) \leqslant M$. 
We say that a set of nodes is infinitely bounded if all the nodes in it are infinitely bounded.

So, we now suppose that there is a constant $R^{\prime} \geqslant R+$ $\max _{v \in \mathcal{S} \cup \mathcal{D}} \operatorname{out}(v)$ and a node $v \in \mathcal{S} \cup \mathcal{D}$ such that $\forall t_{0}$, $\exists t_{1} \geqslant t_{0}$ such that $q_{t}(v) \leqslant R^{\prime}$. We choose an infinitely bounded set $W$, maximal for inclusion, that contains a node in $\mathcal{S} \cup \mathcal{D}$. The size of $W$ is defined as the sum of the queue sizes of the nodes it contains.

Since all nodes in $W$ are infinitely bounded, there is an infinite number of time steps $\left\{t_{i}\right\}_{i \in \mathbb{N}}$ such that the size of $W$ is minimum between $t_{i}$ and $t_{i+1}$, and with $q_{t_{i}}(w) \leqslant R^{\prime}$ for all $w \in W$. The number of stored packets in $W$ at time step $t_{i}-1$ is thus strictly greater than the one at time step $t_{i}: \sum_{w \in W} q_{t_{i}}(w)<\sum_{w \in W} q_{t_{i}-1}(w)$.

The growth of the size of $W$ between time steps $t_{i}-1$ and $t_{i}$ is the following:

$$
\begin{aligned}
\sum_{w \in W} q_{t_{i}-1}(w)= & \sum_{w \in W} q_{t_{i}}(w) \\
& +\sum_{\substack{w \in W\\
}} \text { out }_{t_{i}-1}(w)-\sum_{w \in W} i n_{t_{i}-1}(w) \\
& -\left|\left\{(u, v) \in E_{t_{i}-1}, u \notin W, v \in W\right\}\right| \\
& +\left|\left\{(u, v) \in E_{t_{i}-1}, u \in W, v \notin W\right\}\right|,
\end{aligned}
$$

where $E_{t_{i}-1}$ is the set of links used by LGG at time $t_{i}-1$.

Moreover, $\sum_{w \in W}$ out $_{t_{i}-1}(w) \leqslant \sum_{w \in W}$ out $(w)$ from the definition af the $R$-generalized destinations, and $\sum_{w \in W} i n_{t_{i}-1}(w) \leqslant \sum_{w \in W} i n(w)$ from the assumption made at the beginning of this section. We thus get:

$$
\begin{aligned}
\sum_{w \in W} q_{t_{i}}(w)< & \sum_{w \in W} q_{t_{i}-1}(w)=\sum_{w \in W} q_{t_{i}}(w) \\
& +\sum_{w \in W} \text { out }(w)-\sum_{w \in W} i n(w) \\
& -\left|\left\{(u, v) \in E_{t_{i}-1}, u \notin W, v \in W\right\}\right| \\
& +\left|\left\{(u, v) \in E_{t_{i}-1}, u \in W, v \notin W\right\}\right|
\end{aligned}
$$

which is equivalent to:

$$
\begin{aligned}
\sum_{w \in W} \operatorname{out}(w)> & \sum_{w \in W} \operatorname{in}(w) \\
& +\left|\left\{(u, v) \in E_{t_{i}-1}, u \notin W, v \in W\right\}\right| \\
& -\left|\left\{(u, v) \in E_{t_{i}-1}, u \in W, v \notin W\right\}\right|
\end{aligned}
$$

Since the cut $\left(\left(V \cup\left\{s^{*}\right\}\right),\left\{d^{*}\right\}\right)$ is minimum, $\sum_{w \in W} \operatorname{out}(w)<\sum_{w \in W} i n(w)+|C|$, where $C$ is the set of links incident to $W$ in $G$. So $|C|=\mid\left\{(u, v) \in E_{t_{i}-1}, u \notin\right.$ $W, v \in W\}|+|\left\{(u, v) \in E_{t_{i}-1}, u \in W, v \notin W\right\} \mid$, which leads to:

$$
\begin{aligned}
\sum_{w \in W} \operatorname{out}(w)< & \sum_{w \in W} \operatorname{in}(w) \\
& +\left|\left\{(u, v) \in E_{t_{i}-1}, u \notin W, v \in W\right\}\right| \\
& +\left|\left\{(u, v) \in E_{t_{i}-1}, u \in W, v \notin W\right\}\right|
\end{aligned}
$$

Thus, the number of packets sent at time $t_{i}-1$ from a node in $W$ to a node in $V \backslash W$ is strictly positive. This means that there is a node $w \in V \backslash W$ whose queue length is lower that the one of a node in $W$. It is a contradiction since $W$ was assumed maximum for inclusion.

Whatever $W$ chosen, we find a node in the nodes of $V \backslash$ $W$ infinitely bounded. As $W$ is infinitely bounded and the network size is finite, there is a node $v^{*}$ infinitely bounded such that $W \cup\left\{v^{*}\right\}$ is infinitely bounded. In that way, we show that $V$ is infinitely bounded.

Thus, all nodes in $G$ have a queue of bounded size $\left(q_{t}(v) \leqslant R^{\prime}\right)$ an infinite number of times. Since the number of injected packets at each $t$ is equal to the capacity of extraction of the $R$-generalized sources/destinations, then the number of stored packets in the network never decreases. We therefore conclude that the number of stored packets remains bounded for all $t$.

This concludes the proof of the stability of LGG when the $R$-generalized $\mathcal{S}$-D -network $G$ is saturated at the destinations, assuming Conjecture 1.

\section{Saturated $R$-generalized $\mathcal{S}$-D-network}

Given a $R$-generalized $\mathcal{S}$-D $\mathcal{D}$-network $G$, we show by induction on $|V|$ that LGG is stable on $G$. Our induction hypothesis is the following:

Our protocol $L G G$ is stable on any $R^{\prime}$-generalized $\mathcal{S}^{\prime}$-D $\mathcal{D}^{\prime}$-network of $n$ nodes, $\forall R^{\prime} \geqslant 0, n<|V|$, with $|V|>1$.

In this section, we suppose that there is a minimum cut $(A, B)$ of value $\sum_{v \in \mathcal{S} \cup \mathcal{D}} i n(v)$, such that $|A|,|B|>1$ and $|A|,|B|<|V|$. We sequentially prove that $A$ and $B$ can be viewed as two different generalized networks on which we apply the induction hypothesis to obtain the desired result.

1) The number of packets stored in $B$ is bounded: We show that partition $B$ of the cut $(A, B)$ is a feasible $R$ generalized $\mathcal{S}^{\prime}$ - $\mathcal{D}^{\prime}$-network, and then, that the number of packets stored in $B$ is bounded.

We construct a $\mathcal{S}^{\prime}$-D $\mathcal{D}^{\prime}$-network $B^{\prime}$ that acts as $B$ in $G$. Let $X$ be the set of nodes in $B$ adjacent to a node in partition $A$. Consider the network $B^{\prime}$ containing a set $\mathcal{S}^{\prime}$ of $R$-generalized sources, and a set $\mathcal{D}^{\prime}$ of $R$-generalized destinations, such that $\mathcal{S}^{\prime} \cup \mathcal{D}^{\prime}=X \cup(\mathcal{D} \cap B) \cup(\mathcal{S} \cap B)$, defined in the following way:

- each node in $B \backslash X$ keeps the same behavior in $B^{\prime}$ as the one in $B$ (and thus in $G$ );

- each $v \in X \backslash(\mathcal{S} \cup \mathcal{D})$ becomes a $R$-generalized source of $\mathcal{S}^{\prime}$ with $i_{B^{\prime}}(v)=\left|\Gamma_{\mid A}(v)\right|$, and out $_{B^{\prime}}(v)=0$;

- parameters $i n(v)$ and out $(v)$ of nodes in $X \cap(\mathcal{S} \cup \mathcal{D})$ are updated in $B^{\prime}$ and respectively become $i n_{B^{\prime}}(v)=$ $\operatorname{in}(v)+\left|\Gamma_{\mid A}(v)\right|$, and $\operatorname{out}_{B^{\prime}}(v)=\operatorname{out}(v)$. If $\operatorname{in}_{B^{\prime}}(v) \geqslant$ out $_{B^{\prime}}(v)$, then $v \in \mathcal{S}^{\prime}$, otherwise $v \in \mathcal{D}^{\prime}$.

By induction hypothesis, if the $R$-generalized $\mathcal{S}^{\prime}-\mathcal{D}^{\prime}$ network $B^{\prime}$ is feasible, then LGG is stable on $B^{\prime}$. Then, by definition of $B^{\prime}$, we can inject packets into nodes of $X$ in such a way that $B^{\prime}$ acts as $B$ in $G$. Therefore, if LGG is stable on $B^{\prime}$, then it is stable on $B$. It remains to prove that $B^{\prime}$ is feasible.

Since $(A, B)$ has value $\sum_{v \in \mathcal{S} \cup \mathcal{D}} i n(v)$, each link on the border of the cut transmit one unit of flow $\Phi$. By definition of a $R$-generalized source and by well choosing the characteristics of the nodes of $X$, the flow $\Phi_{B^{\prime}}$ that injects 
in $(v)$ packets in each $R$-generalized source/destination $v \in \mathcal{S} \cup \mathcal{D}$, and that follows links used by $\Phi$ in $G$ is feasible. Indeed, from the Kirchoff laws respected by the flow, $\Phi_{B^{\prime}}$ is lower than or equal to $\Phi$ on each link used by $\Phi$.

We have found a feasible flow $\Phi_{B^{\prime}}$ in $B^{\prime}$, thus the $R$ generalized $\mathcal{S}^{\prime}$-D $\mathcal{D}^{\prime}$-network $B^{\prime}$ is feasible, and then the number of stored packets in $B$ is bounded. Let $R_{B}$ be the maximum number of packets stored in $B$.

2) The number of packets stored in $A$ is bounded: We use the same reasoning to show that the number of stored packets in partition $A$ is bounded. $A$ can be viewed as a $R_{B}$-generalized $\mathcal{S}^{\prime \prime}$ - $\mathcal{D}^{\prime \prime}$-network $A^{\prime}$ in which $\mathcal{S}^{\prime \prime} \cup \mathcal{D}^{\prime \prime}=$ $Y \cup(\mathcal{D} \cap A) \cup(\mathcal{S} \cap A)$, with $Y$ the set of nodes in $A$ adjacent to some node in $B$. We then prove that $A^{\prime}$ is feasible in order to bound the number of stored packets in it.

Sets $\mathcal{S}^{\prime \prime}$ and $\mathcal{D}^{\prime \prime}$ of $R_{B}$-generalized sources and destinations are defined in the following way:

- each node in $A \backslash Y$ keeps the same behavior $A^{\prime}$ as in $A$ (and then in $G$ );

- each $v \in Y \backslash(\mathcal{S} \cup \mathcal{D})$ becomes a $R_{B^{-}}$generalized destination of $\mathcal{D}^{\prime \prime}$ with $\operatorname{out}_{A^{\prime}}(v)=\left|\Gamma_{\mid B}(v)\right|$, and $i n_{A^{\prime}}(v)=0$;

- finally, the parameters $i n(v)$ and $\operatorname{out}(v)$ of nodes $v \in$ $Y \cap(\mathcal{S} \cup \mathcal{D})$ are updated in $A^{\prime}$ and become respectively $\operatorname{in}_{A^{\prime}}(v)=\operatorname{in}(v)$, and $\operatorname{out}_{B^{\prime}}(v)=\operatorname{out}(v)+\left|\Gamma_{\mid B}(v)\right|$. If $\operatorname{in}_{A^{\prime}}(v) \geqslant \operatorname{out}_{A^{\prime}}(v)$, then $v \in \mathcal{S}^{\prime \prime}$, otherwise $v \in \mathcal{D}^{\prime \prime}$.

Remark 2: Remark that $\mathcal{D}^{\prime \prime} \neq \emptyset$. Indeed, if it not the case, then $\sum_{v \in(\mathcal{S} \cup \mathcal{D})} i n(v)>\sum_{v \in Y}\left|\Gamma_{\mid B}(v)\right|=$ $\sum_{v \in(\mathcal{S} \cup \mathcal{D})} \Phi\left(s^{*}, v\right)$ and we obtain a contradiction according to the existence of a feasible flow $\Phi$ in $G$.

This remark allows us to apply the induction hypothesis and to conclude that if the $R_{B}$-generalized $\mathcal{S}^{\prime \prime}$ - $\mathcal{D}^{\prime \prime}$-network $A^{\prime}$ is feasible, then LGG is stable on $A^{\prime}$. Then, as LGG is stable on $A^{\prime}$ and that $Y$ has the same behavior in $A^{\prime}$ and in $A$, the stability of LGG on $A$ is proved. As for $B$, the flow $\Phi$ restricted to the nodes in $A^{\prime}$ is a feasible flow in $A^{\prime}$ extended. Therefore, the number of packets stored in $A$ remains bounded.

\section{Conclusion}

We showed that LGG is stable in a $\mathcal{S}$-D-network in which the number of injected packets at each time step is lower than or equals to the value of a maximum flow in the network, assuming Conjecture 1 when the flow is constrained at the destination nodes. This work opens several perspectives and points out some conjectures on the stability of queueing systems.

Considering the initial case of a classical $\mathcal{S}$ - $\mathcal{D}$-network, If the arrival rate changes at each time step, we conjecture a weaker feasibility condition.

Conjecture 2: If the number of packets generated at some time step $t$ exceeds the available capacity, i.e. the value of a maximum flow, then it is sufficient and necessary that some interval of time latter the number of packets generated is low enough to extracts the excess.
More formally, let $G$ be a $\mathcal{S}$-D-network, $\Phi$ a maximum $s^{*}-d^{*}$-flow in $G^{*}$ with value $f^{*}$, and $i n_{t}(s)$ the number of injected packets in the queue of a source $s \in \mathcal{S}$ at time $t$. The stability condition would thus be:

$$
\begin{gathered}
\text { For all } t \text { and } d t \text {, if } \sum_{s \in \mathcal{S}} \sum_{k=1}^{d t} \text { in } n_{t+k}(s)>d t \cdot f^{*} \text {, then } \\
\text { there is a time } t^{\prime} \text { such that } \\
\sum_{s \in \mathcal{S}} \sum_{k=1}^{t^{\prime}} i n_{t+k}(s) \leqslant\left(t^{\prime}-t\right) f^{*} .
\end{gathered}
$$

If we now consider the case where the arrival rate in the network follows a uniform distribution, then we conjecture the following result:

Conjecture 3: If the number of injected packets $i n_{t}(s)$ at time $t$ in the queue of source $s \in \mathcal{S}$ follows a uniform distribution of mean strictly less than than the value of a minimum $\mathcal{S}$-D -cut, then with high probability our protocol is stable on the $\mathcal{S}$ - $\mathcal{D}$-network.

The case of a dynamic network in which the topology (nodes and links) changes among time, is an important perspective of research [5]. The stability of LGG in these networks might depend on the existence of a feasible flow in the network. In other words:

Conjecture 4: If the number of injected packets ensures the existence of a feasible $\mathcal{S}$ - $\mathcal{D}$-flow, then LGG is stable on the network, at least in the unsaturated case.

For addressing more practical situations in autonomic networking, one should get rid of the assumption, made in this work, that there is no interference among simultaneous transmissions. In order to deal with wireless interferences, we have to compute, for each step of our algorithm LGG, the set of pairwise compatible links $E_{t}$. The goal is to find, at each time step $t$, the optimal set $E_{t}$ in order to guaranty that the number of stored packets in the $\mathcal{S}$ - $\mathcal{D}$-network remains bounded:

Conjecture 5: If an oracle can provide an optimal set $E_{t}$ in the $\mathcal{S}$-D -network $G$ at time $t$, then LGG is stable on $G$.

\section{REFERENCES}

[1] D. Peleg, Distributed computing: a locality-sensitive approach. Philadelphia, PA, USA: Society for Industrial and Applied Mathematics, 2000.

[2] X. Wu and R. Srikant, "Regulated maximal matching: A distributed scheduling algorithm for multi-hop wireless networks with node-exclusive spectrum sharing," in 44th IEEE Conference on Decision and Control, and the European Control Conference (CDC-ECC), Dec. 2005, pp. 5342-5347.

[3] L. Tassiulas and A. Ephremides, "Stability properties of constrained queueing systems and scheduling policies for maximum throughput in multihop radio networks," IEEE Transactions on Automatic Control, vol. 37, no. 12, pp. 1936-1948, Dec. 1992.

[4] P. Tsaparas, "Stability in adversarial queueing theory," Master Thesis, Department of Computer Science, University of Toronto, Tech. Rep., 1997.

[5] B. Awerbuch, P. Berenbrink, A. Brinkmann, and C. Scheideler, "Simple routing strategies for adversarial systems," in FOCS, 2001

[6] A. Goldberg and R. Tarjan, "A new approach to the maximumflow problem," Journal of the Association for Computing Machinery, vol. 35, no. 4, pp. 921-940, Oct. 1988.

[7] F. Huc, C. Molle, N. Nisse, S. Perennes, and H. Rivano, "Stability of a local greedy distributed routing algorithm," INRIA, Tech. Rep. RR-6871, Mar. 2009. 


\section{AnNeXe}

In this section, we include the omitted proof of Properties 3 and 6 , which we recall bellow.

Property 5: Given an unsaturated $R$-generalized $\mathcal{S}$-D $\mathcal{D}$ network, when running LGG, the growth of the network state $P_{t}$ between two consecutive steps stays bounded for all $t$ :

$$
\begin{aligned}
P_{t+1}-P_{t} \leqslant & 2|\mathcal{S} \cup \mathcal{D}|\left(R+\text { out }_{\text {max }}\right) \text { out }_{\text {max }} \\
& +\Delta^{2}(3 n-2|\mathcal{S} \cup \mathcal{D}|)+4|\mathcal{S} \cup \mathcal{D}| \Delta R,
\end{aligned}
$$

where out max $_{\text {max }}=\max _{v \in \mathcal{S} \cup \mathcal{D}}$ out $(v)$.

Proof: From equation 1, we seek to upper bound $\delta_{t}$. At each time step $t$, a $R$-generalized source/destination $v$ in $G$ injects $i n_{t}(v) \leqslant i n(v)<\Phi\left(s^{*}, v\right)$ packets in its queue. Similarly, $v$ extracts out $_{t}(v) \leqslant \min \left\{\right.$ out $\left.(v), q_{t}(v)\right\}$ packets of its queue, with out $(v) \geqslant \min \left\{\right.$ out $\left.(v), q_{t}(v)\right\}$ if $q_{t}(v)>R \geqslant \Delta$, and $\operatorname{out}_{t}(v) \geqslant 0$ otherwise.

In this condition, the first change occurs in equation 3 which becomes:

$$
\begin{aligned}
\delta_{t}=\sum_{(u, v) \in E_{t}}\left(q_{t}(v)-q_{t}(u)\right) & +\sum_{s \in \mathcal{S}} q_{t}(s)\left(\text { in }_{t}(s)-\text { out }_{t}(s)\right) \\
& -\sum_{d \in \mathcal{D}} q_{t}(d)\left(\text { out }_{t}(d)-i n_{t}(d)\right) .
\end{aligned}
$$

We now compare the value of the sum of the difference of the queue sizes on the links used by LGG and those following a maximum flow. As in Section III, we decompose the sum into three sums over $E_{t}^{\Phi}, E_{t}^{\Phi} \backslash E_{t}$, and $E_{t} \backslash E_{t}^{\Phi}$. The generalization of the network does not change the behaviour of the flow. Nevertheless, as $R$-generalized sources and destinations both inject and extract packets, we get a different equation 4 :

$$
\begin{aligned}
\sum_{(u, v) \in E_{t}^{\Phi}} & \left(q_{t}(v)-q_{t}(u)\right) \\
& =\sum_{d \in \mathcal{D}} q_{t}(d)\left(\Phi\left(d, d^{*}\right)-\Phi\left(s^{*}, d\right)\right) \\
& -\sum_{s \in \mathcal{S}} q_{t}(s)\left(\Phi\left(s^{*}, s\right)-\Phi\left(s, d^{*}\right)\right) \\
& =\sum_{v \in \mathcal{S} \cup \mathcal{D}} q_{t}(v)\left(\Phi\left(v, d^{*}\right)-\Phi\left(s^{*}, v\right)\right)
\end{aligned}
$$

By definition of LGG, $q_{t}(v)-q_{t}(u)<0$ for $e=(u, v) \in$ $E_{t} \backslash E_{t}^{\Phi}$, except if $u \in \mathcal{S} \cup \mathcal{D}$ or $v \in \mathcal{S} \cup \mathcal{D}$ lies on its queue size. Suppose that $v$ is lying, then $q_{t}(u) \leqslant q_{t}(v) \leqslant R$. But $v$ reveals a queue size $q_{t}^{\prime}(v) \leqslant q_{t}(u)$, so we bound the difference: $q_{t}(v)-q_{t}(u) \leqslant R$. Suppose now that $v$ is not lying, then $u$ is lying and declares a height $R \geqslant q_{t}^{\prime}(u) \geqslant$ $q_{t}(v)$, which also bounds the difference $q_{t}(v)-q_{t}(u)$ by $R$.

So for each neighbour $u$ of $v \in \mathcal{S} \cup \mathcal{D}$, the difference $q_{t}(v)-q_{t}(u)$ is upper bounded by $R$, and so is the difference for each $v$ neighbour of $u \in \mathcal{S} \cup \mathcal{D}$, leading to the following inequality:

$$
\sum_{(u, v) \in E_{t} \backslash E_{t}^{\Phi}}\left(q_{t}(v)-q_{t}(u)\right) \leqslant 2|\mathcal{S} \cup \mathcal{D}| \Delta R .
$$

Considering the links of the flow that are not used by LGG: $e=(u, v) \in E_{t}^{\Phi} \backslash E_{t}$, only the case where $q_{t}(v)-q_{t}(u) \leqslant 0$ matters (it contributes positively to $\sum_{(u, v) \in E_{t}}\left(q_{t}(v)-q_{t}(u)\right)$ that we want to bound). If a link $(u, v)$ such that $q_{t}(v) \leqslant q_{t}(u)$ is not used by LGG, it means that:

- $q_{t}(u) \leqslant \Delta$ and $u$ has already sent all its packets to its neighbours of smaller height. Then, the difference $q_{t}(v)-q_{t}(u)$ is lower bounded by $-\Delta$ as we saw in Section III.

- $u \in \mathcal{S} \cup \mathcal{D}$ lies on its queue size. Therefore $q_{t}(v) \leqslant$ $q_{t}(u) \leqslant R$, but $u$ declare a height $q_{t}^{\prime}(u)<q_{t}(v)$. Then $q_{t}(v)-q_{t}(u) \geqslant q_{t}(v)-R \geqslant-R$.

- $u \in \mathcal{S} \cup \mathcal{D}$ does not lie. So $v$ lies and declare $R \geqslant$ $q_{t}^{\prime}(v)>q_{t}(u)$. The difference $q_{t}(v)-q_{t}(u)$ is always lower bounded by $-R$.

Thus, the difference of the queue sizes $q_{t}(v)-q_{t}(u)$ is lower bounded by $-R$ if $u \in \mathcal{S} \cup \mathcal{D}$ or $v \in \mathcal{S} \cup \mathcal{D}$, and by $-\Delta$ otherwise, leading to the following lower bound:

$\sum_{(u, v) \in E_{t}^{\Phi} \backslash E_{t}}\left(q_{t}(v)-q_{t}(u)\right) \geqslant-\Delta^{2}(n-2|\mathcal{S} \cup \mathcal{D}|)-2|\mathcal{S} \cup \mathcal{D}| \Delta R$.

We then put these bounds into the difference of the queue sizes on links of LGG and obtain:

$$
\begin{aligned}
\sum_{(u, v) \in E_{t}}\left(q_{t}(v)-q_{t}(u)\right) \leqslant & \sum_{v \in \mathcal{S} \cup \mathcal{D}} q_{t}(v)\left(\Phi\left(v, d^{*}\right)-\Phi\left(s^{*}, v\right)\right) \\
& +\Delta^{2}(n-2|\mathcal{S} \cup \mathcal{D}|)+4|\mathcal{S} \cup \mathcal{D}| \Delta R
\end{aligned}
$$

Then, for $\delta_{t}$ (Eq. 6), we finally get:

$$
\begin{aligned}
\delta_{t} \leqslant \sum_{\substack{v \in \mathcal{S} \cup \mathcal{D}\\
}} q_{t}(v)\left(\left(\Delta^{2}(n-2|\mathcal{S} \cup \mathcal{D}|)+4|\mathcal{S} \cup \mathcal{D}| \Delta R\right.\right.
\end{aligned}
$$

From this equation, we deduce some properties:

- $\forall s \in \mathcal{S}, \Phi\left(s^{*}, s\right)>i n(s)$ since the $R$-generalized $\mathcal{S}$ - $\mathcal{D}$ network is unsaturated, and $i n(s) \geqslant i n_{t}(s)$ from the definition of a $R$-generalized source. Then, $i n_{t}(v)-$ $\Phi\left(s^{*}, v\right)<0$ for all $v \in \mathcal{S}$.

- $\forall d \in \mathcal{D}, \Phi\left(s^{*}, d\right) \geqslant i n(d)$ from the definition of a feasible network.

- $\forall v \in \mathcal{S} \cup \mathcal{D}$ : if $\Phi\left(v, d^{*}\right) \leqslant$ out $_{t}(d)$, then

$$
\delta_{t} \leqslant \Delta^{2}(n-2|\mathcal{S} \cup \mathcal{D}|)+4|\mathcal{S} \cup \mathcal{D}| \Delta R .
$$

On the contrary, if $\Phi\left(v, d^{*}\right)>$ out $_{t}(d)$, then $q_{t}(v) \leqslant$ $R+\operatorname{out}(v)$. Indeed, if $q_{t}(v)>R+\operatorname{out}(v)$, then out $_{t}(v) \geqslant \min \left\{q_{t}(v)-R\right.$, out $\left.(v)\right\}=\operatorname{out}(v) \geqslant \Phi\left(v, d^{*}\right)$, which leads to a contradiction. Thus,

$$
\begin{aligned}
\delta_{t} & \leqslant \sum_{v \in \mathcal{S} \cup \mathcal{D}}(R+\operatorname{out}(v)) \operatorname{out}(v) \\
& +\Delta^{2}(n-2|\mathcal{S} \cup \mathcal{D}|)+4|\mathcal{S} \cup \mathcal{D}| \Delta R \\
& \leqslant|\mathcal{S} \cup \mathcal{D}|\left(R+\max _{v \in(\mathcal{S} \cup \mathcal{D})} \operatorname{out}(v)\right) \max _{v \in(\mathcal{S} \cup \mathcal{D})} \operatorname{out}(v) \\
& +\Delta^{2}(n-2|\mathcal{S} \cup \mathcal{D}|)+4|\mathcal{S} \cup \mathcal{D}| \Delta R
\end{aligned}
$$

We have derived an upper bound for $\delta_{t}$ that is independent of $t$. From inequality 2, we deduce that the growth of the 
network's state $P_{t}$ between two consecutive steps is upper bounded.

We now prove the second unproved property:

Property 6: Given an unsaturated $R$-generalized $\mathcal{S}$ - $\mathcal{D}$ network running LGG, given a constant $Y$ large enough, if $P_{t}>n Y^{2}$, at step $t+1$, the number of stored packets in the network strictly decreases:

$$
\begin{aligned}
P_{t+1}-P_{t}<\quad & -2|\mathcal{S} \cup \mathcal{D}|\left(R+\text { out }_{\text {max }}\right) \text { out }_{\text {max }} \\
& -\Delta^{2}(3 n-2|\mathcal{S} \cup \mathcal{D}|)-4|\mathcal{S} \cup \mathcal{D}| \Delta R,
\end{aligned}
$$

where out $t_{\text {max }}=\max _{v \in \mathcal{S} \cup \mathcal{D} \text { out }}(v)$.

Proof: Let $A=2|\mathcal{S} \cup \mathcal{D}|\left(R+\right.$ out $\left._{\max }\right)$ out $t_{\max }+\Delta^{2}(3 n-$ $2|\mathcal{S} \cup \mathcal{D}|)+4|\mathcal{S} \cup \mathcal{D}| \Delta R$. From inequality 2, proving Property 6 is equivalent to show that, if $P_{t} \geqslant n Y^{2}$, then $\delta_{t}<-\frac{A+n \Delta^{2}}{2}$. As in Section III, the proof follows two cases.

First, suppose that it exists a generalized node $x \in \mathcal{S} \cup \mathcal{D}$ such that

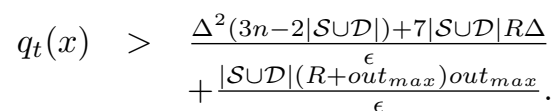

Recall that $\epsilon=\min _{v \in \mathcal{S} \cup \mathcal{D}}\left(\Phi\left(s^{*}, v\right)-i n(v)\right)$, then from equation 7 and the fact that $i n_{t}(v)-\Phi\left(s^{*}, v\right) \leqslant i n(v)-$ $\Phi\left(s^{*}, v\right)<-\epsilon$ for all $v \in \mathcal{S} \cup \mathcal{D}$ and for $\epsilon>0$, we get as in Section III:

$$
\begin{aligned}
\delta_{t} & \leqslant-\epsilon q_{t}(s)+\Delta^{2}(n-|\mathcal{S} \cup \mathcal{D}|)+5|\mathcal{S} \cup \mathcal{D}| \Delta R \\
& <-\frac{A+n \Delta^{2}}{2}
\end{aligned}
$$

which proves the first part of Property 6 .

Then, we have $\forall v \in \mathcal{S} \cup \mathcal{D}$

$$
q_{t}(v) \leqslant \frac{\Delta^{2}(3 n-2|\mathcal{S} \cup \mathcal{D}|)+7|\mathcal{S} \cup \mathcal{D}| R \Delta}{\epsilon} .
$$

In this case, if $P_{t} \geqslant n Y^{2}$, then it exists a node $x \in$ $V \backslash(\mathcal{S} \cup \mathcal{D})$ with large height and a path defined as in Section III. The sum of the difference of the queue sizes along this path is thus: $\sum_{i<k, q_{t}\left(u_{i}\right)>q_{t}\left(u_{i+1}\right)}\left(q_{t}\left(u_{i+1}\right)-\right.$ $\left.q_{t}\left(u_{i}\right)\right) \leqslant q_{t}\left(u_{k}\right)-q_{t}(x)$.

Recall that for all $e=(u, v) \in E_{t}$ with either $v \notin \mathcal{S} \cup \mathcal{D}$, or $v \in \mathcal{S} \cup \mathcal{D}$ and $q_{t}(v)>R$ (respectively with either $u \notin \mathcal{S} \cup \mathcal{D}$, or $u \in \mathcal{S} \cup \mathcal{D}$ and $q_{t}(u)>R$ ), we have $q_{t}(v)-$ $q_{t}(u)<0$. Moreover, for all $e=(u, v) \in E_{t}$ such that $v \in \mathcal{S} \cup \mathcal{D}$ and $q_{t}(v) \leqslant R$ (respectively $u \in \mathcal{S} \cup \mathcal{D}$ and $q_{t}(u) \leqslant R$ ), we have $q_{t}(v)-q_{t}(u) \leqslant R$.

The sum along links used by LGG is thus bounded:

$$
\sum_{(u, v) \in E_{t}}\left(q_{t}(v)-q_{t}(u)\right) \leqslant 2|\mathcal{S} \cup \mathcal{D}| \Delta R+q_{t}\left(u_{k}\right)-q_{t}(x) .
$$

We also get the following lower bound:

$$
\sum_{d \in \mathcal{D}} q_{t}(d)\left(\text { out }_{t}(d)-i n_{t}(d)\right) \geqslant \sum_{d \in \mathcal{D}} q_{t}(d)-(R+1) f^{*}
$$

because if $q_{t}(d) \leqslant R$ for a $R$-generalized destination $d$, then out $_{t}(d) \leqslant i n_{t}(d)+1$ by definition. Moreover, by definition of the value of the maximum $s^{*}$ - $d^{*}$-flow $f^{*}$, $\sum_{d \in \mathcal{D}} i n_{t}(d) \leqslant f^{*}$.
From equation 6 , we therefore get:

$$
\begin{aligned}
\delta_{t} \leqslant & \sum_{s \in \mathcal{S}} q_{t}(s)\left(\text { in }_{t}(s)-\text { out }_{t}(s)\right)+(R+1) f^{*} \\
& -\sum_{d \in \mathcal{D}} q_{t}(d)\left(\text { out }_{t}(d)-i n_{t}(d)\right) \\
& +2(|\mathcal{S} \cup \mathcal{D}|) \Delta R+q_{t}\left(u_{k}\right)-q_{t}(x) \\
\leqslant & \sum_{s \in \mathcal{S}} q_{t}(s)\left(\text { in }_{t}(s)-\text { out }_{t}(s)\right)+(R+1) f^{*} \\
& +2|\mathcal{S} \cup \mathcal{D}| \Delta R-q_{t}(x) .
\end{aligned}
$$

By choosing $Y$ sufficiently large such that $q_{t}(x)>Y$, Property 6 is satisfied. 